\title{
Three-dimensional magnetic flux rope structure formed by multiple sequential $X$-line reconnection at the magnetopause
}

\author{
J. Zhong, ${ }^{1}$ Z. Y. Pu, ${ }^{1}$ M. W. Dunlop, ${ }^{2}$ Y. V. Bogdanova, ${ }^{2,3}$ X. G. Wang, ${ }^{4}$ C. J. Xiao, ${ }^{4}$ \\ R. L. Guo, ${ }^{1}$ H. Hasegawa, ${ }^{5}$ J. Raeder, ${ }^{6}$ X. Z. Zhou, ${ }^{7}$ V. Angelopoulos, ${ }^{7}$ Q. G. Zong, ${ }^{1}$ \\ S. Y. Fu, ${ }^{1}$ L. Xie, ${ }^{1}$ M. G. G. T. Taylor, ${ }^{8}$ C. Shen, ${ }^{9}$ J. Berchem, ${ }^{7}$ Q. H. Zhang, ${ }^{10}$ \\ M. Volwerk, ${ }^{11}$ and J. P. Eastwood ${ }^{12}$
}

Received 28 November 2012; revised 18 March 2013; accepted 16 April 2013; published 28 May 2013.

[1] On 14 June 2007, four Time History of Events and Macroscale Interactions during Substorms spacecraft observed a flux transfer event (FTE) on the dayside magnetopause, which has been previously proved to be generated by multiple, sequential X-line reconnection (MSXR) in a 2-D context. This paper reports a further study of the MSXR event to show the 3-D viewpoint based on additional measurements. The 3-D structure of the FTE flux rope across the magnetospheric boundary is obtained on the basis of multipoint measurements taken on both sides of the magnetopause. The flux rope's azimuthally extended section is found to lie approximately on the magnetopause surface and parallel to the X-line direction; while the axis of the magnetospheric branch is essentially along the local unperturbed magnetospheric field lines. In the central region of the flux rope, as distinct from the traditional viewpoint, we find from the electron distributions that two types of magnetic field topology coexist: opened magnetic field lines connecting the magnetosphere and the magnetosheath and closed field lines connecting the Southern and Northern hemispheres. We confirm, therefore, for the first time, the characteristic feature of the 3-D reconnected magnetic flux rope, formed through MSXR, through a determination of the field topology and the plasma distributions within the flux rope. Knowledge of the complex geometry of FTE flux ropes will improve our understanding of solar wind-magnetosphere interaction.

Citation: Zhong, J., et al. (2013), Three-dimensional magnetic flux rope structure formed by multiple sequential X-line reconnection at the magnetopause, J. Geophys. Res. Space Physics, 118, 1904-1911, doi:10.1002/jgra.50281.

\section{Introduction}

[2] It is widely accepted that magnetic flux transfer events (FTEs), identified as bipolar signatures in the normal magnetic field component $\left(\mathrm{B}_{\mathrm{N}}\right)$ to the Earth's magnetopause

\footnotetext{
${ }^{1}$ School of Earth and Space Sciences, Peking University, Beijing, China

${ }^{2}$ Rutherford-Appleton Laboratory, Oxfordshire, UK.

${ }^{3}$ Mullard Space Science Laboratory, University College London, Dorking, UK.

${ }^{4}$ School of physics, Peking University, Beijing, China.

${ }^{5}$ Institute of Space and Astronautical Science, JAXA, Sagamihara, Japan.

${ }^{6}$ Space Science Center and Physics Department, University of New Hampshire, Durham, USA.

${ }^{7}$ Institute of Geophysics and Planetary Physics, University of California, Los Angeles, California, USA.

${ }^{8}$ ESTEC, ESA, Noordwijk, Netherlands.

${ }^{9}$ State Key Laboratory of Space Weather, NSSC, CAS, Beijing, China.

${ }^{10}$ SOA Key Laboratory for Polar Science, Polar Research Institute of China, Shanghai, China.

${ }^{11}$ Space Research Institute, Austrian Academy of Sciences, Graz, Austria.

${ }^{12}$ Blackett Laboratory, Imperial College London, London, UK.

Corresponding author: Z. Y. Pu, School of Earth and Space Sciences, Peking University, 5 Yiheyuan St., Haidian, Beijing 100871, China. (zypu@pku.edu.cn)

(C)2013. American Geophysical Union. All Rights Reserved. 2169-9380/13/10.1002/jgra.50281
}

(MP) [Russell and Elphic, 1978], are transient signatures of moving flux ropes created during periods of magnetic reconnection (MR). Such flux ropes form a channel between the magnetosphere and magnetosheath to allow transport of solar wind plasma and energy into geospace [Elphic, 1995; Lui et al., 2008; Paschmann et al., 1982; Zhang et al., 2008]. Several MR-related models have been proposed to explain the flux rope structure and the formation mechanism [Lee and Fu, 1985; Liu and Hu, 1988; Pu et al., 1990; Raeder, 2006; Russell and Elphic, 1978; Scholer, 1988]. It has been suggested that MR can originate at multiple sites, resulting in complex 3-D structures highly sensitive to the ambient conditions. Clear observations of flux rope structures therefore require in situ measurements taken by multiple spacecraft at well positioned locations [Sibeck et al., 2008; Zhang et al., 2012], which are extremely rare.

[3] An FTE on the dayside MP was observed to pass by the Time History of Events and Macroscale Interactions during Substorms (THEMIS) spacecraft constellation on 14 June 2007 [Dunlop et al., 2011] and has been interpreted [Hasegawa et al., 2010] within a 2-D picture of multiple sequential X-line reconnection (MSXR) [Raeder, 2006], where the FTE signature is consistent with being created between two $\mathrm{X}$ lines on the MP, one 

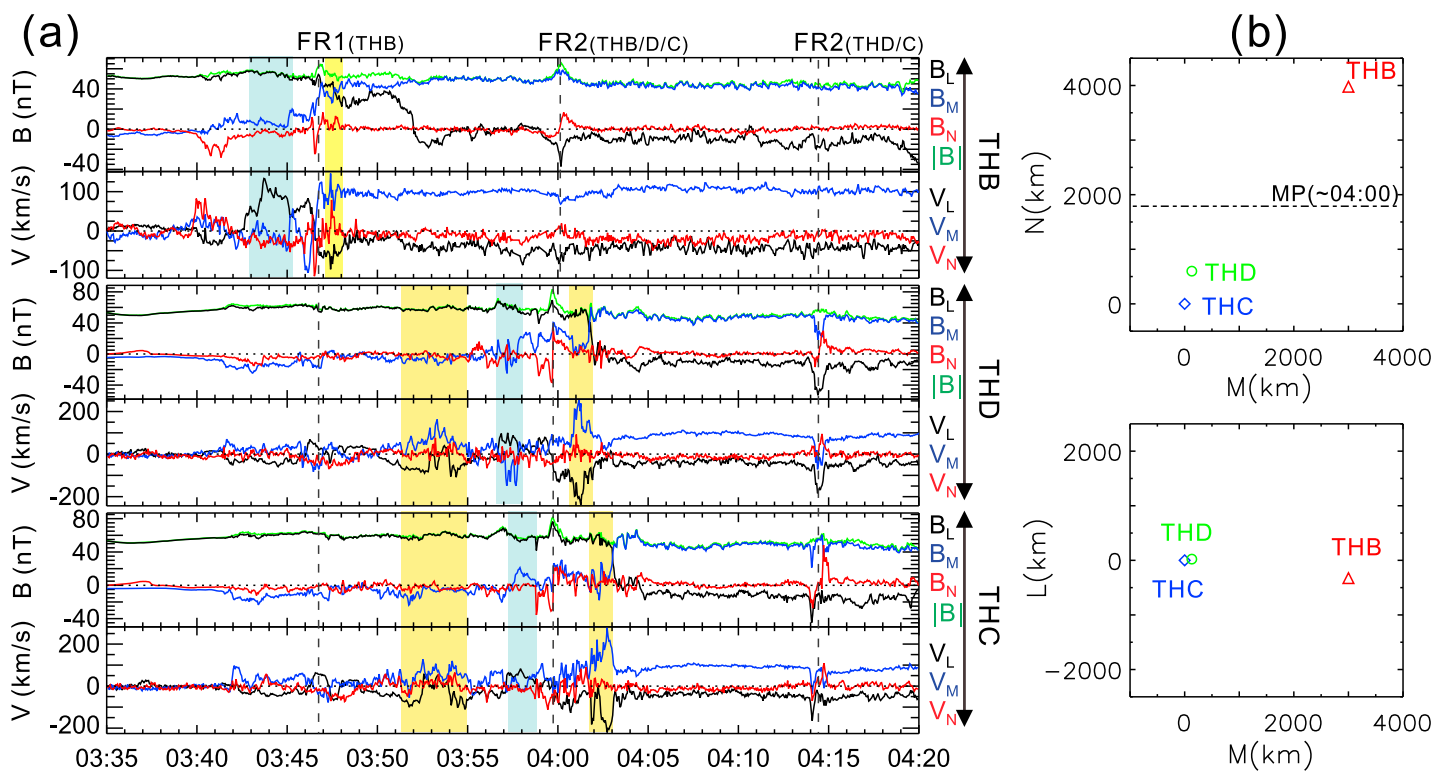

Figure 1. (color). (a) Data from three THEMIS probes (B, D and C) on 14 June 2007, from 03:35 to 04:20UT. Magnetic field and plasma bulk velocity are plotted in LMN coordinates. Three flux ropes (FR, marked by vertical dashed lines) are encountered sequentially. (b) Relative positions of three THEMIS spacecraft in the LMN coordinate system. The location of the reference spacecraft THC was $(10.2,3.7,-2.3) \mathrm{R}_{\mathrm{E}}$ in GSM.

on the south-dusk side and another forming later on the subsolar side. In Hasegawa et al. [2010] two X lines were inferred from two oppositely directed flows converging toward the FTE, based on a 2-D Grad-Shafranov (G-S) reconstruction of the magnetospheric branch of the flux rope. This 2-D picture, however, remained incomplete because key 3-D structures are not described: closed field loops in the 2-D map cannot display the complicated 3-D magnetic field topology, nor the orientation of the $\mathrm{X}$ lines, which were not directly observed in the G-S reconstruction. In fact, previous observations have indicated that FTEs are 3-D phenomena with complex structures and geometry [Louarn et al., 2004; Owen et al., 2008]. The MR process producing flux ropes should also be $3-\mathrm{D}$ in nature [Daughton et al., 2011; Fu et al., 1990; Lee et al., 1993; Øieroset et al., 2011; Tan et al., 2011]. It is then highly desirable to investigate the flux rope geometry as 3-D structures. Such an investigation may help to understand plasma and energy transport across the MP and is thus of essential importance for understanding solar wind-magnetosphere coupling. In this paper, the event is revisited to infer the large-scale 3-D structure of the flux rope and field line topology, placing its axis-orientation and motion in context, based on the analysis of data from the electrostatic analyzers (ESA) [McFadden et al., 2008] and Fluxgate Magnetometers (FGM) [Auster et al., 2008] onboard the THEMIS spacecraft.

\section{Observations of the Flux Rope and 3-D Analysis}

\subsection{Overview of the Event}

[4] The event (see Figure1a) occurred during 03:40-04:20 UT on 14 June 2007, on the southern, postnoon quadrant of the subsolar magnetopause. During the interval, the interplanetary magnetic field was strongly duskward. Figurela shows that the magnetic field and ion velocity transformed into the local boundary normal coordinates (LMN) [Russell and Elphic, 1979], where $\mathbf{N}$ points outward along the normal of the local MP and L (M) lies in the MP tangential surface pointing northward (duskward). The $\mathbf{N}$ is derived from minimum variance analysis of the MP crossing by THEMIS-B (THB); the $\mathbf{M}$ is determined by $\mathbf{z} \times \mathbf{N}$, where $\mathbf{z}$ denotes the $\mathbf{z}$ axis of the GSM coordinate system; The $\mathbf{L}$ forms the right-hand system with the $\mathbf{N}$ and $\mathbf{M}$ : i.e., $\mathbf{N} \times \mathbf{M}$. The $\mathbf{L}, \mathbf{M}$ and $\mathbf{N}$ unit vectors in the GSM coordinates are $(0.123,0.040,0.992),(-0.313,0.950,0)$, and $(0.942,0.310,-0.129)$, respectively.

[5] Three FTE signatures (negative-positive $B_{N}$ bipolar), interpreted as MR flux ropes and marked by vertical dashed lines in Figure 1a, are observed when the spacecraft were slowly exiting from the magnetosphere into the magnetosheath. Figure 1b shows the positions of THEMIS-C (THC) and THEMIS-D (THD) relative to THB, with a cut through the MP at the time of the central event. THB was ahead of THD (THC) by about 3377 (3976) $\mathrm{km}$ in the $N$-direction and first crossed the MP at $\sim 03: 47$ UT. About $15 \mathrm{~min}$ later, THD and THC sequentially crossed the MP at 04:01:46 UT and 04:02:57 UT. Thus, THB encountered the first (second) flux ropes and THC/THD the second (third) ones on the magnetospheric (magnetosheath) sides of the MP.

[6] During the crossings of the first and second flux ropes, two oppositely directed ion jets converging toward the flux ropes were clearly detected, consistent with these flux ropes being created through MSXR process, as confirmed in Hasegawa et al. [2010] for the second one. In Hasegawa et al., [2010], the observational evidence for MSXR are obtained based on the fact that particle signatures of reconnection on both south-dusk and subsolar 
Table 1. Results of Principal Axis Analysis ${ }^{\mathrm{a}}$

\begin{tabular}{|c|c|c|c|c|c|c|}
\hline FTEs & S/C (MVA Time Interval) & $\frac{\text { Eigen Ratio }}{(\lambda 2 / \lambda 3)}$ & $\begin{array}{l}\text { Axis Orientation } \\
(\mathrm{LMN})\end{array}$ & $\alpha_{\text {MSP-line }}$ & $\alpha_{\mathrm{X} \text {-line }}$ & $\begin{array}{l}\text { Crossing FTE } \\
\text { Section }\end{array}$ \\
\hline$\sim 03: 47 \mathrm{UT}$ & THB (03:46:10-03:47:18) & 5 & $(0.985,0.172,-0.010)$ & $14.4^{\circ}$ & $41.6^{\circ}$ & magnetospheric arm \\
\hline \multirow[t]{2}{*}{$\sim 04: 00 \mathrm{UT}$} & THC (03:59:24-04:00:40) & 3 & $(0.982,0.162,0.099)$ & $14.0^{\circ}$ & $42.1^{\circ}$ & magnetospheric arm \\
\hline & THB (03:59:16-04:01:58) & 5 & $(0.515,0.853,-0.084)$ & $63.4^{\circ}$ & $7.3^{\circ}$ & azimuthally extended \\
\hline \multirow[t]{2}{*}{$\sim 04: 14$ UT } & THC (04:13:54-04:15:00) & 21 & $(0.631,0.753,-0.186)$ & $55.5^{\circ}$ & $1.4^{\circ}$ & azimuthally extended \\
\hline & THD (04:14:10-04:14:45) & 38 & $(0.666,0.737,-0.114)$ & $52.8^{\circ}$ & $3.6^{\circ}$ & azimuthally extended \\
\hline
\end{tabular}

\footnotetext{
${ }^{\mathrm{a}} \alpha_{\mathrm{MSP}-\text { line }}$ is defined as the angle between the axis orientation and the local, unperturbed magnetospheric field; $\alpha_{\mathrm{X}-\text {-line }}$ is defined as the angle between the
} axis orientation and the $\mathrm{X}$ line.

sides of the FTE were observed by THB, and that the subsolar $\mathrm{X}$ line was shown to become active later than the south-dusk side $\mathrm{X}$ line. Furthermore, before the first and between the first and second flux ropes, THB and THC/THD observed a south-duskward $\left(-V_{\mathrm{L}}, V_{\mathrm{M}}\right.$, shaded in yellow in Figure 1a) and then north-dawnward $\left(V_{\mathrm{L}}\right.$, $V_{\mathrm{M}}$, shaded in green in Figure 1a) jet reversal, respectively. Similar jet reversals have been interpreted as the $\mathrm{X}$ line passing by the spacecraft [Pu et al., 2005]. Around the third flux rope, the plasma jets were not observed, due to the spacecraft entering into the magnetosheath far away from the MP.

\subsection{Orientations of the Flux Rope and its 3-D Structure}

[7] Determining the flux ropes axis orientation is critical for revealing their 3-D structures. In literatures, the magnetic-field-based minimum variance analysis (BMVA) technique [Sonnerup and Cahill, 1967; Sonnerup and Scheible, 1998] has been widely used for analyzing the axial direction of flux ropes [Fear et al., 2012, and references therein]. Xiao et al. [2004] made a numerical MVA test for different flux rope models and found that the relationship between the axis orientation and the directions of the eigenvectors of BMVA were critically dependent on the spacecraft paths relative to the flux ropes and structure of the flux rope encountered. They showed that the BMVA can provide a valuable technique to infer the flux rope axis in two cases. One is that the spacecraft passes through the center of the flux rope. Regarding the internal field of the flux rope as magnetic force-free configuration [Goldstein, 1983; Lepping et al., 1990], one can find that the intermediate variance direction of B from BMVA best fits the axial orientation [Lepping et al., 1990], while for non-force-free flux ropes, the BMVA might in some situations fail as the useful tool [Xiao et al., 2004]. Another case is that the spacecraft trajectory remains sufficient outside the flux rope (i.e., "grazing" FTE), the BMVA presents a unique tool for inferring the flux rope orientation, with the minimum variance direction being best representing the axial direction [Farrugia et al., 1987].

[8] For the second flux rope on 14 June 2007, the GradShafranov reconstruction showed that THC/THD was passing through near its center [Hasegawa et al., 2010]; we thus could take the intermediate BMVA direction to approximate the orientation of the section of the flux rope encountered by THC/THD. On the other hand, THB detected magnetosheath plasma properties during most of the $\mathrm{B}_{\mathrm{N}}$ bipolar variation, which are commonly regarded as that the spacecraft is crossing the draping region of the FTE on the magnetosheath side
[Le et al., 1999]. Therefore, it is reasonable to assume that the minimum variance direction of $\mathbf{B}$ from BMVA can best represent the local flux rope axial orientation crossed by THB.

[9] For THC, the calculated flux rope orientation is $(0.982,0.162,0.099)$ in $\mathrm{LMN}$, very close $\left(\sim 3.7^{\circ}\right)$ to the normal to the G-S plane: $(0.971,0.224,0.085)_{\mathrm{LMN}}$ [Hasegawa et al., 2010]. Thus, it is reasonable to adopt $(0.982,0.162,0.099)_{\mathrm{LMN}}$ as the axial orientation for the section of flux rope observed by $\mathrm{THC}$, which is nearly aligned to the local magnetospheric magnetic field direction. This is consistent with that the flux rope encountered by THC is the part of the magnetospheric arm of the flux rope. On the other hand, the calculated orientation of the local flux rope encountered by THB is $(0.515,0.853$, $0.084)_{\text {LMN }}$ (see Table 1). This direction is dominantly in the $\mathbf{M}$ direction and deviates from the MP tangential surface by only about $4.8^{\circ}$, indicating that this section of the flux rope is lying nearly along the local MP surface (L-M plane). We therefore regard it as the azimuthally extended section of the flux rope. Note that THB is separated from THD by 3001 (3377) $\mathrm{km}$ in the $\mathbf{M}(\mathbf{N})$ direction (Figure 1b), therefore it samples a different part of the flux rope in comparison with that encountered by THC.

[10] Furthermore, the orientation of the expected $\mathrm{X}$ line can be calculated using the component merging model [Sonnerup, 1974]. The component merging model predicts that the average magnetic fields on two sides of the MP current sheet have a common component parallel to the $\mathrm{X}$ line (this also defines the direction of maximum current arising from the crossed magnetic fields). The averaged adjacent magnetospheric and magnetosheath magnetic fields ( $\left.\mathbf{B}_{\mathbf{M S P}}, \mathbf{B}_{\mathbf{M S H}}\right)$ can thus be used to calculate the X-line orientation: $\mathbf{N} \times\left(\mathbf{B}_{\mathbf{M S P}}-\mathbf{B}_{\mathbf{M S H}}\right) /\left|\mathbf{N} \times\left(\mathbf{B}_{\mathbf{M S P}}-\mathbf{B}_{\mathbf{M S H}}\right)\right|$. The $\mathbf{B}_{\mathbf{M S P}}$ and $\mathbf{B}_{\mathbf{M S H}}$ are, respectively, $52 \times(0.997,-0.074$, $0.037) \mathrm{nT}$ and $47 \times(-0.234,0.972,0.003) \mathrm{nT}$ in LMN, averaged over the time period of 03:35:00-03:39:00 UT and 04:06:00-04:11:00 UT, respectively. Then, the calculated $\mathrm{X}$-line orientation is $\sim(0.62,0.78,0.00)_{\mathrm{LMN}}$, nearly parallel to the orientation of the flux rope section encountered by THB, with the angle between them being $\sim 7.3^{\circ}$ in the MP surface. This is also consistent with the fact that THB encountered the azimuthally extended section of the flux rope near the MP. The orientations of the parts of the first and third flux ropes encountered by THEMIS probes, and the angle they make with respect to the local, unperturbed magnetospheric field $\left(\alpha_{\text {MSP-line }}\right)$ and to the $X$ line $\left(\alpha_{X-\text { line }}\right)$ are listed in Table 1. 
(a)

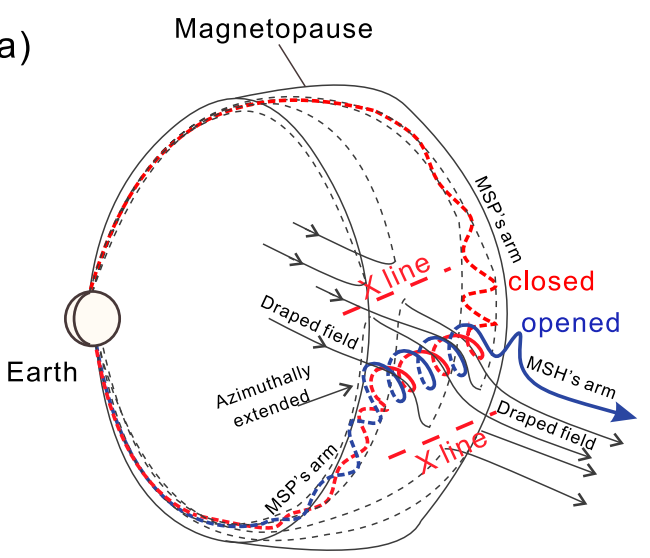

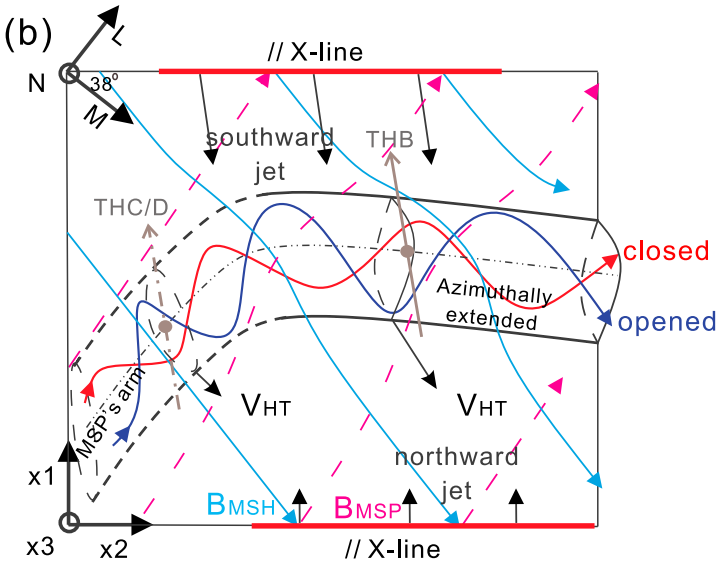

(b)

// X-line

Figure 2. (color). Schematic diagrams of 3-D geometry of the second FTE: (a) Large-scale view from the dawnside. The azimuthally extended flux rope is approximately lying in the MP surface and parallel to the $\mathrm{X}$ lines; the magnetospheric (magnetosheath) branch of the flux rope connects the azimuthally extended part to the North/South Hemisphere (magnetosheath) and approximately parallel to the local magnetospheric (magnetosheath) field. (b) Detailed structures near the observational site of the FTE. THB crossed the magnetospheric arm, and THC/THD crossed the azimuthally extended section. Coexistence of open and closed field line topologies was observed inner the flux rope.

[11] Figure 2a summarizes the observations of the second flux rope and surrounding regions in a 3-D largescale picture where the axis of the magnetosheath branch of the flux rope is assumed to be close to the magnetosheath field direction. Figure $2 b$ shows an interpretation of the results obtained in this section, in which the direction of the $\mathrm{X}$ line, the orientations of the magnetospheric arm, and the azimuthally extended section of the flux rope are plotted. We will return to these two figures later in the later sections.

\subsection{Electron Energy-Pitch Angle Spectrum and 3-D Field Line Geometries}

[12] The presence of 3-D feature of the flux rope geometry is confirmed by the electron distribution functions taken by THC during the second flux rope crossing. The electron energy-pitch angle spectrum measured by THC through the whole flux rope crossing is shown in Figure $3 \mathrm{a}$. From top to bottom are shown: the magnetic field magnitude and LMN components; energy-time spectrogram, and three pitch angle-time spectrograms for three different energy ranges corresponding to a typical magnetospheric (3394-30333 eV), accelerated magnetosheath $(495-1130 \mathrm{eV})$ and magnetosheath $(42-215 \mathrm{eV})$ population, respectively. The electron energy-pitch angle spectrum shows that the reconnected flux rope possesses a complex and variable substructure.

[13] The magnetospheric electron distribution (above $3394 \mathrm{eV}$ ) is used here to identify the magnetic topology of the flux rope (between two vertical dashed lines in Figure 3a). Around 03:59:53 UT (the segment covered by blue bars in Figure 3a), predominantly field-aligned $\left(0-90^{\circ}\right.$; out-flowing) magnetospheric electrons were observed, suggesting an open field line geometry connecting the southern magnetosphere to the magnetosheath. Meanwhile, during the majority of time period (the segment covered by red bars in Figure 3a) isotropic magnetospheric electrons were observed. These are signatures of closed magnetic field lines connecting both hemispheres [Bogdanova et al., 2008].

[14] The typical electron velocity distributions in the open and closed flux region are shown as 2-D cuts in Figures $3 \mathrm{~b}$ and $3 \mathrm{c}$, respectively. The behaviors of the magnetosheath and accelerated magnetosheath populations agree well with the field line topology interpretation. At 03:59:52-03:59:55 (Figure 3b), low-energy magnetosheath $(<100 \mathrm{eV})$ electrons are seen to enter into the magnetosphere antiparallel to the open field lines $\left(90^{\circ}-180^{\circ}\right)$; whereas at 03:59:40-03:59:43 UT (Figure 3c), low-energy magnetosheath and accelerated magnetosheath populations are clearly found to be trapped on the closed field lines (bistreaming).

[15] The THC data here show that both closed and open field lines coexist in the flux rope core region. Moreover, THD detected similar signatures inside the magnetospheric arm in the event studied (not shown); the first and third flux ropes have such a feature as well. Such coexistence of both open and closed field line topologies within the same flux rope might be a common aspect in many 3-D MSXR events (see Figure 2a). We will return to this point later.

\subsection{Flux Rope Motion in 3-D View}

[16] To further pursue the nature of the MSXR formation of flux ropes, we apply de Hoffmann-Teller (HT) analysis [Sonnerup et al., 1987] to study the motion of the second flux rope. The HT frame determination is performed based on the measured ion bulk velocities and magnetic field data. A steadily accelerating HT frame (linear time-variation of the frame velocity) is considered here. It is found that good accelerating HT frames existed for periods corresponding to the leading and trailing edge crossings by THB and THD. Results are listed in Table 2. The velocity $\mathbf{V}_{\mathbf{H T}}$ and acceleration $\mathbf{a}_{\mathbf{H T}}$ are given in $\mathbf{x 1}$, $\mathbf{x 2}$, x3 components (the rotated LMN coordinates, as shown on Figure 2a, where $\mathbf{x} \mathbf{3}$ is in the direction of 

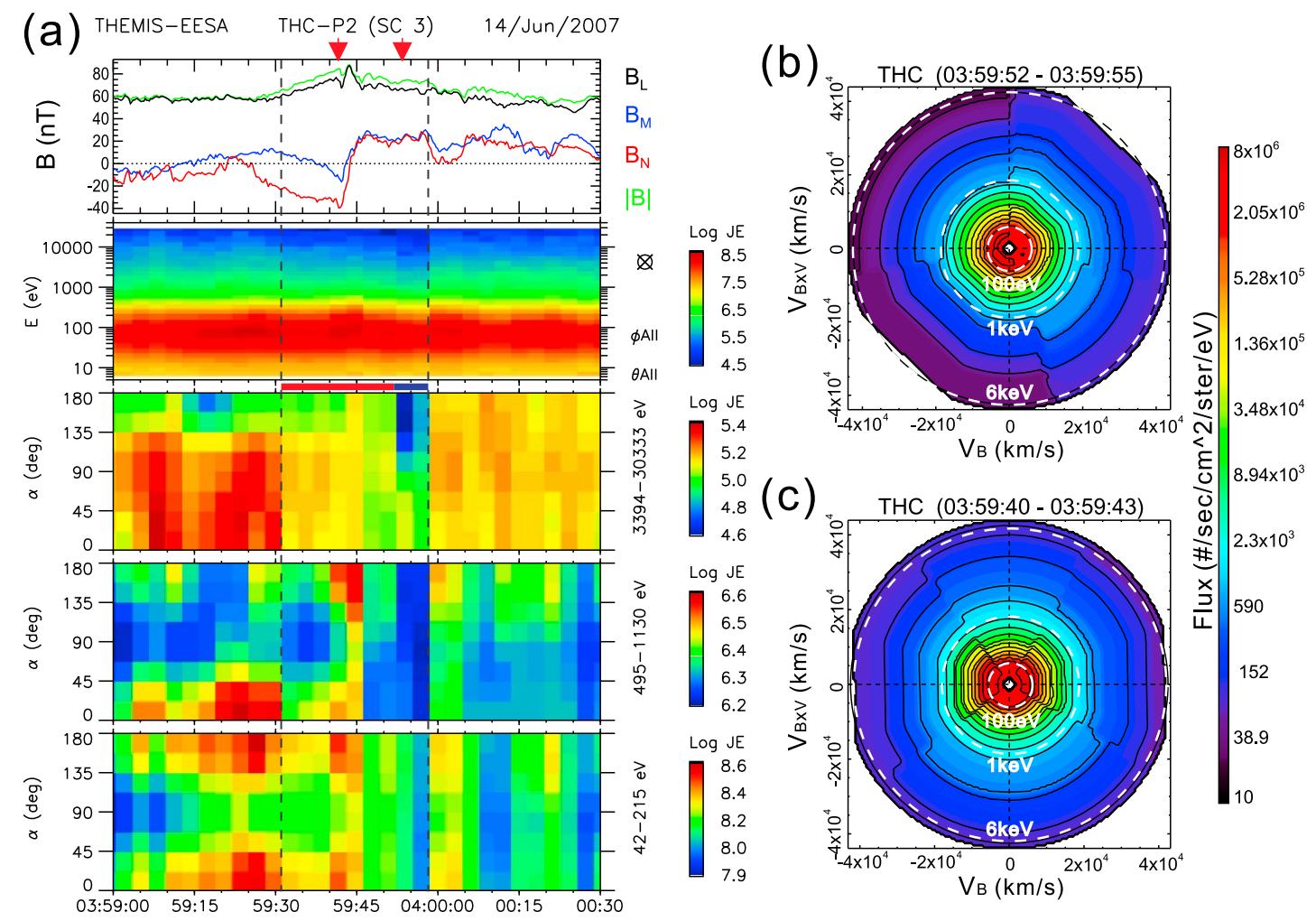

Figure 3. (color). (a) Energy and pitch-angle spectrum of electrons measured by THC when the spacecraft was crossing the second FTE: from top to button, the magnetic field magnitude, $\mathrm{B}_{\mathrm{N}}, \mathrm{B}_{\mathrm{M}}$ and $\mathrm{B}_{\mathrm{L}}$ components; electron energy spectra; the pitch angle spectrogram for three energy ranges indicated on the left (indicating magnetospheric, accelerated magnetosheath and magnetosheath populations respectively). Each subpanel shows data averaged over $3 \mathrm{~s}$ (spin resolution). (b and c) Three seconds averaged electron velocity distributions measured by THC for two time periods. The $X$-axis represents the component parallel to the magnetic field; the $Y$-axis corresponds to the component in the direction of $\mathbf{v} \times \mathbf{B}$ where $\mathbf{v}$ is the electron bulk velocity. The color coding corresponds to the electron particle flux. Three white circle dash lines indicate $100 \mathrm{eV}, 1 \mathrm{keV}$, and $6 \mathrm{keV}$ respectively.

$\mathbf{N}, \mathbf{x} 2$ is parallel to the $X$ line and $\mathbf{x} 1$ completes the triad). The four excellent correlation coefficients cc $(\approx 1)$ and very small ratios $D\left(\mathbf{V}_{\mathbf{H T}}\right) / D(0)$ (see Table 2 ) confirm that the ion frozen-in condition holds and one can readily identify a steadily accelerating HT frame for each time interval of interest.

[17] The azimuthally extended flux rope is bounded in the draped magnetosheath region. At the beginning of the leading edge and the end of the trailing edge, THB was located in the draped magnetosheath field area. The observed magnetosheath flow was $(-74,89,-24) \mathrm{km} / \mathrm{s}$ in LMN. As shown in Table 2, for the azimuthally extended section of the second FTE, $\boldsymbol{V}_{\mathbf{H T}}$ at the beginning of the leading edge and the end of the trailing edge are almost the same and are close to the flow velocity in the draped magnetosheath field region. The calculated HT velocity here is thus reasonable and believable. Furthermore, at the center of the $\mathrm{B}_{\mathrm{N}}$ bipolar, THB grazed the azimuthally extended flux rope for a short time. At the end of the leading edge and the beginning of the trailing edge, the spacecraft was traveling inside the azimuthally extended section of the flux rope, the calculated HT velocities are $(-155,99,11)$ and $(-143$, $96,22) \mathrm{km} / \mathrm{s}$, respectively, and they are also close to each other. Thus, we take the average of these two inner $\boldsymbol{V}_{\mathbf{H T}}$ values, i.e., $(-149,98,17) \mathrm{km} / \mathrm{s}$, to approximate the velocity of the azimuthally extended flux rope section. One thus sees

Table 2. Steadily Accelerating HT Frame Test for FTE $2^{\mathrm{a}}$

\begin{tabular}{|c|c|c|c|c|c|c|}
\hline Interval & $\mathrm{cc}$ & $D\left(\mathbf{V}_{\mathbf{H T}}\right) / D(0)$ & Slope & $\mathrm{a}_{\mathrm{HT}}\left(\mathrm{km} / \mathrm{s}^{2}\right)$ & $\boldsymbol{V}_{\mathbf{H T}} \operatorname{Start}(\mathrm{km} / \mathrm{s})$ & $\boldsymbol{V}_{\mathbf{H T}}$ End $(\mathrm{km} / \mathrm{s})$ \\
\hline B-L (03:59:48-04:00:08) & 0.996 & 0.003 & 0.96 & $(-4.0,1.3,0.7)$ & $(-76,74,-2)$ & $(-155,99,11)$ \\
\hline B-Т (04:00:08-04:00:34) & 0.981 & 0.037 & 1.02 & $(2.5,-0.5,-0.9)$ & $(-143,96,22)$ & $(-79,84,-3)$ \\
\hline D-L (03:59:20-03:59:41) & 0.995 & 0.008 & 0.97 & $(-4.6,2.6,-0.1)$ & $(52,8,-35)$ & $(-45,62,-38)$ \\
\hline D-T (03:59:41-04:00:02) & 0.996 & 0.006 & 1.00 & $(-4.0,-2.0,0.2)$ & $(-59,49,-33)$ & $(-142,8,-30)$ \\
\hline
\end{tabular}

${ }^{\mathrm{a}} \mathrm{L}=$ leading edge, $\mathrm{T}=$ trailing edge, $\mathrm{B}=\mathrm{THB}, \mathrm{D}=\mathrm{THD}$. cc is the correlation coefficient between three components of the convection electric field and HT electric field; $D\left(\mathbf{V}_{\mathbf{H T}}\right) / D(0)$ is the mean square ratio of the residual electric field in the HT frame to the induced electric field in the spacecraft frame, which measures the quality of the HT frame. The latter three columns are expressed in the rotated LMN coordinates (x1, $\mathbf{x} 2, \mathbf{x} 3)$, where $\mathbf{x} 3$ points to the $\mathbf{N}$ direction, $\mathbf{x} 2$ is parallel to the $\mathrm{X}$ line (positive west-northward) and $\mathbf{x 1}=\mathbf{x} \mathbf{3} \times \mathbf{x} \mathbf{2}$. 

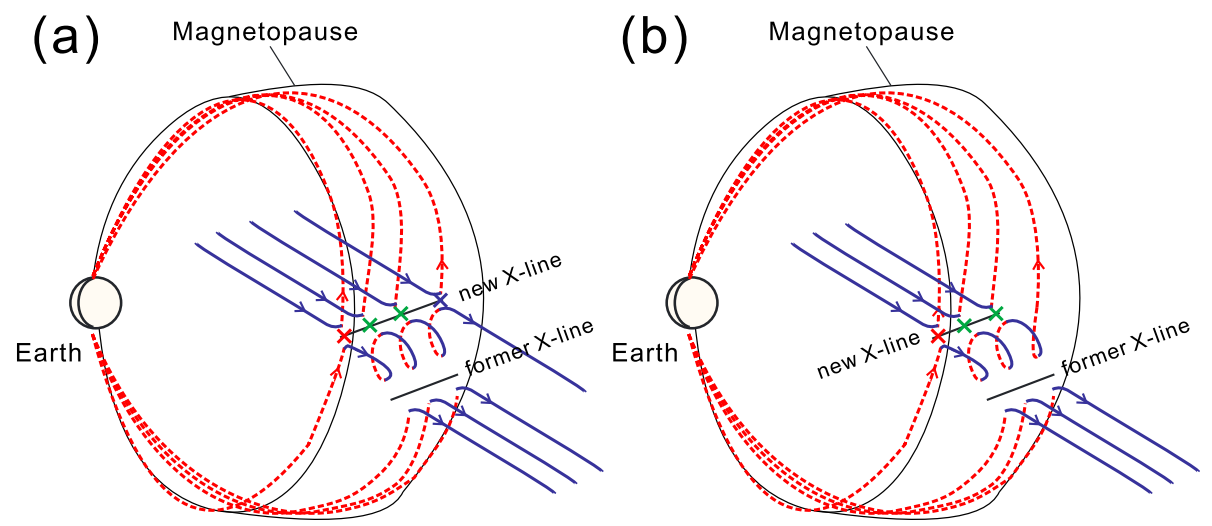

Figure 4. (color). The configurations of the (a) open and (b) closed flux rope on the magnetopause when the new X line starts to form near the subsolar region. The dashed red curves represent the field lines initially on the magnetospheric side, and the solid blue curves represent the field lines initially on the magnetosheath side. The black lines indicate $\mathrm{X}$ lines. The red, blue and green crosses denote the locations at the new X line where an open field line (which links the magnetosheath to the Northern Hemisphere) reconnected with a magnetospheric, magnetosheath field line and intersected with another open field line, respectively.

that it is accelerating southward and westward, mainly perpendicular to the local rope axis.

[18] Similarly, for the magnetospheric arm of the flux rope, we can take the average of the $\boldsymbol{V}_{\mathbf{H T}}$ at the end of the leading edge and the beginning of the trailing edge, i.e., $(-52,56,-36) \mathrm{km} / \mathrm{s}$ as its approximate velocity and see that it is accelerating southward, westward, and tailward. Note that the flux rope's azimuthally extended section is moving faster than the magnetospheric arm.

\section{Discussion and Summary}

[19] In this paper, using the THEMIS electron distribution data in combination with the magnetic field measurements, we showed that two types of magnetic field topology were observed inside the reconnected flux ropes: the opened field lines connecting the southern magnetosphere and the magnetosheath, and closed field lines connecting both hemispheres. We interpret the coexistence of these two magnetic connection types as the result of 3-D MSXR at the magnetopause and infer that it may manifest a common feature in the MSXR process. Coexistence of both open and closed field line topologies within the same flux rope has been seen in 3-D simulations of multiple X-line reconnection [Fu et al., 1990; Lee et al., 1993; Tan et al., 2011], and now has been shown to exist in the THEMIS measurements. Contrary to the traditional view on the open geometry of FTE flux ropes [Øieroset et al., 2011], we here, for the first time, show observations of the mixed topology inside the FTE flux rope.

[20] Magnetopause MR with one X line at low latitudes produces open field lines connecting the magnetosheath to the northern (southern) magnetosphere [Gosling et al., 1990]. However, in the ongoing MSXR, when the former $\mathrm{X}$ line has swept poleward/flankward by the magnetosheath flow and a new $\mathrm{X}$ line appears in the subsolar region, helical field lines are created to form a flux rope. As can be seen in Figure 4, field lines with two ends connecting to the magnetosheath and magnetosphere, respectively, create an open geometry (Figure 4a); while those with both ends connecting to the magnetosphere constitute a closed configuration (Figure $4 \mathrm{~b}$ ). The open flux rope continuously provides a channel for transport of flux and plasmas across the magnetopause; while the closed-field structures, converted from the open structures, may make significant contributions to the formation of the low latitude boundary layer on closed geomagnetic field lines [Boudouridis et al., 2002]. We suppose that such complex magnetic field topologies inside the flux ropes via MSXR might also appear in reconnection processes in the interplanetary current sheet [Moldwin et al., 2000; Ruan et al., 2009], and in other planetary magnetospheres [Huddleston et al., 1997; Russell and Walker, 1985; Slavin et al., 2010; Walker and Russell, 1985], where in situ measurements are not as many as in the terrestrial case.

[21] Additionally, using data from multiple spacecraft located on both sides of the MP, we were able to infer the 3-D structure of the flux rope. The detailed analysis results of the orientation and motion of the second flux rope are shown in the Figure $2 \mathrm{~b}$. The azimuthally extended part of the flux rope and its magnetospheric arm are clearly shown. It directly confirms the presence of double X lines, parallel to, and on either side of, the azimuthally extended part of the flux rope. The different motion between the azimuthally extended section and the magnetospheric arm of the flux rope implies that the overall shape of the flux rope will be changed during evolution of the FTEs. The computed orientations of the first and third flux ropes are consistent with the geometry inferred for the second flux rope (see Table 1). THB crossed the magnetospheric arm of the first flux rope and azimuthally extended section of the second flux rope in succession; THC/THD remotely grazed the first flux rope and then encountered the magnetospheric arm of the second and azimuthally extended part of the third flux rope. Such long-time observations have shown a whole picture of the consecutive formation of flux ropes near the subsolar region during MSXR. 
[22] In summary, we have shown, for the first time, the observational 3-D features of FTE flux ropes resulting from MSXR: i.e., (a) the 3-D structure of the FTE flux rope across the magnetospheric boundary, based on multiple satellite measurements made on both sides of the magnetopause; (b) the coexistence of both open and closed field lines inside the central region of the FTE flux ropes; and (c) FTEs make important contributions to formation of the magnetospheric boundary layer of closed field lines. Knowledge of the 3-D structure and complex geometry of FTE flux ropes will improve our understanding of solar wind-magnetosphere interaction. Up to now, generation of FTEs and 3-D structure of FTE flux ropes is not fully understood yet. Further in situ 3-D measurements and 3-D high-resolution global simulations are highly desired.

[23] Acknowledgments. The study was supported by the NSFC Grants 41274167, 40731056, 40974095, 41004072 and 10975012, the Chinese National Key Basic Research Science Foundation (2011CB811400), and the Doctoral Fund of the Ministry of Education of China (20090001110012). The study was initiated from a working group sponsored by ISSI, Berne, Switzerland. We acknowledge NASA contract NAS5-02099 for the use of data from the THEMIS Mission, C. W. Carlson and J. P. McFadden for use of ESA data, and K. H. Glassmeier, U. Auster and W. Baumjohann for use of FGM data.

\section{References}

Auster, H. U., et al. (2008), The THEMIS Fluxgate Magnetometer, Space Sci. Rev., 141, 235-264.

Bogdanova, Y. V., et al. (2008), Formation of the low-latitude boundary layer and cusp under the northward IMF: Simultaneous observations by Cluster and Double Star, J. Geophys. Res., 113, A07S07, doi:10.1029/ 2007JA012762.

Boudouridis, A., H. E. Spence, and T. G. Onsager (2002), A new look at the pulsed reconnection model of the dayside magnetopause, Advances in Global Magnetospheric Structure, Dynamics, and Region Coupling, Proceedings, 30(10), 2295-2300.

Daughton, W., V. Roytershteyn, H. Karimabadi, L. Yin, B. J. Albright, B. Bergen, and K. J. Bowers (2011), Role of electron physics in the development of turbulent magnetic reconnection in collisionless plasmas, Nat. Phys., 7(7), 539-542.

Dunlop, M. W., et al. (2011), Extended Magnetic Reconnection across the Dayside Magnetopause, Phys. Rev. Lett., 107(2), 025004.

Elphic, R. C. (1995), Observations of flux transfer events: A review, in Physics of the Magnetopause, edited, AGU, Washington, DC, pp. 225-233.

Farrugia, C. J., R. C. Elphic, D. J. Southwood, and S. W. H. Cowley (1987), Field and flow perturbations outside the reconnected field line region in flux transfer events: Theory, Planet. Space Sci., 35(2), 227-240.

Fear, R. C., S. E. Milan, and K. Oksavik (2012), Determining the axial direction of high-shear flux transfer events: Implications for models of FTE structure, J. Geophys. Res., 117, A09220, doi:10.1029/2012JA017831.

Fu, Z. F., L. C. Lee, and Y. Shi (1990), A three-dimensional MHD simulation of the multiple $\mathrm{X}$ line reconnection process, Physics of magnetic flux ropes (A92-31201 12-75).

Goldstein, H. (1983), On the field configuration of a magnetic cloud, in Solar Wind Five, edited by M. Neugebauer, NASA Conf. Publ., Washington, D. C., CP-2280, 731-733.

Gosling, J. T., M. F. Thomsen, S. J. Bame, R. C. Elphic, and C. T. Russell (1990), Plasma-Flow Reversals at the Dayside Magnetopause and the Origin of Asymmetric Polar-Cap Convection, J. Geophys. Res., 95(A6), 8073-8084.

Hasegawa, H., et al. (2010), Evidence for a flux transfer event generated by multiple X-line reconnection at the magnetopause, Geophys. Res. Lett., 37, L16101, doi:10.1029/2010GL044219.

Huddleston, D. E., C. T. Russell, G. Le, and A. Szabo (1997), Magnetopause structure and the role of reconnection at the outer planets, $J$ Geophys. Res., 102(A11), 24289-24302.

Le, G., J. T. Gosling, C. T. Russell, R. C. Elphic, M. F. Thomsen, and J. A. Newbury (1999), The magnetic and plasma structure of flux transfer events, J. Geophys. Res., 104(A1), 233-245.

Lee, L. C., and Z. F. Fu (1985), A Theory of Magnetic-Flux Transfer at the Earths Magnetopause, Geophys. Res. Lett., 12(2), 105-108.
Lee, L. C., Z. W. Ma, Z. F. Fu, and A. Otto (1993), Topology of Magnetic Flux Ropes and Formation of Fossil Flux Transfer Events and Boundary Layer Plasmas, J. Geophys. Res., 98(A3), 3943-3951.

Lepping, R. P., J. A. Jones, and L. F. Burlaga (1990), Magnetic field structure of interplanetary magnetic clouds at $1 \mathrm{AU}, J$. Geophys. Res., 95(A8), $11957-11965$

Liu, Z. X., and Y. D. Hu (1988), Local Magnetic Reconnection Caused by Vortices in the Flow Field, Geophys. Res. Lett., 15(8), 752-755.

Louarn, P., A. Fedorov, E. Budnik, G. Fruit, J. A. Sauvaud, C. C. Harvey, I. Dandouras, H. Reme, M. C. Dunlop, and A. Balogh (2004), Cluster observations of complex 3D magnetic structures at the magnetopause, Geophys. Res. Lett., 31, L19805, doi:10.1029/2004GL020625.

Lui, A. T. Y., D. G. Sibeck, T. Phan, V. Angelopoulos, J. McFadden, C. Carlson, D. Larson, J. Bonnell, K. H. Glassmeier, and S. Frey (2008), Reconstruction of a magnetic flux rope from THEMIS observations, Geophys. Res. Lett., 35, L17S05, doi:10.1029/2007GL032933.

McFadden, J. P., C. W. Carlson, D. Larson, M. Ludlam, R. Abiad, B. Elliott, P. Turin, M. Marckwordt, and V. Angelopoulos (2008), The THEMIS ESA Plasma Instrument and In-flight Calibration, Space Sci. Rev., 141(1-4), 277-302.

Moldwin, M. B., S. Ford, R. Lepping, J. Slavin, and A. Szabo (2000), Smallscale magnetic flux ropes in the solar wind, Geophys. Res. Lett., 27(1), 57-60.

Øieroset, M., et al. (2011), Direct Evidence for a Three-Dimensional Magnetic Flux Rope Flanked by Two Active Magnetic Reconnection X Lines at Earth's Magnetopause, Phys. Rev. Lett., 107(16), 165007.

Owen, C. J., A. Marchaudon, M. W. Dunlop, A. N. Fazakerley, J. M. Bosqued, J. P. Dewhurst, R. C. Fear, S. A. Fuselier, A. Balogh, and H. Reme (2008), Cluster observations of "crater" flux transfer events at the dayside high-latitude magnetopause, J. Geophys. Res., 113, A07S04, doi:10.1029/2007JA012701.

Paschmann, G., G. Haerendel, I. Papamastorakis, N. Sckopke, S. J. Bame, J. T. Gosling, and C. T. Russell (1982), Plasma and Magnetic Field Characteristics of Magnetic Flux Transfer Events, J. Geophys. Res., 87(A4), 2159-2168.

Pu, Z. Y., M. Yei, and Z. X. Liu (1990), Generation of Vortex-Induced Tearing Mode Instability at the Magnetopause, J. Geophys. Res., 95(A7), 10559-10566.

$\mathrm{Pu}, \mathrm{Z}$. Y., et al. (2005), Double Star TC-1 observations of component reconnection at the dayside magnetopause: a preliminary study, Ann. Geophys.-Germany, 23(8), 2889-2895.

Raeder, J. (2006), Flux Transfer Events: 1. generation mechanism for strong southward IMF, Ann. Geophys., 24(1), 381-392.

Ruan, P., A. Korth, E. Marsch, B. Inhester, S. Solanki, T. Wiegelmann, Q. G. Zong, R. Bucik, and K. H. Fornacon (2009), Multiple-spacecraf study of an extended magnetic structure in the solar wind, J. Geophys. Res., 114, A02108, doi:10.1029/2008JA013769.

Russell, C. T., and R. C. Elphic (1978), Initial ISEE Magnetometer Results: Magnetopause Observations, Space Sci. Rev., 22(6), 681-715.

Russell, C. T., and R. C. Elphic (1979), ISEE Observations of FluxTransfer Events at the Dayside Magnetopause, Geophys. Res. Lett., $6(1), 33-36$.

Russell, C. T., and R. J. Walker (1985), Flux Transfer Events at Mercury, J. Geophys. Res., 90(A11), 11067-11074.

Scholer, M. (1988), Magnetic flux transfer at the magnetopause based on single X line bursty reconnection, Geophys. Res. Lett., 15(4), 291-294

Sibeck, D. G., M. Kuznetsova, V. Angelopoulos, K. H. Glassmeier, and J. P. McFadden (2008), Crater FTEs: Simulation results and THEMIS observations, Geophys. Res. Lett., 35, L17S06, doi:10.1029/ 2008GL033568.

Slavin, J. A., et al. (2010), MESSENGER observations of large flux transfer events at Mercury, Geophys. Res. Lett., 37, L02105, doi:10.1029/ 2009GL041485

Sonnerup, B. U. Ö. (1974), Magnetopause Reconnection Rate, J. Geophys. Res., 79(10), 1546-1549.

Sonnerup, B. U. Ö., and L. J. Cahill (1967), Magnetopause Structure and Attitude from Explorer 12 Observations, J. Geophys. Res., 72(1), $171-183$.

Sonnerup, B. U. Ö., and M. Scheible (1998), Minimum and maximum variance analysis, in Analysis Methods for Multi-Spacecraft Data, edited by G. Paschmann, and P. W. Daly, 185-220, Int. Space Sci. Inst., Bern, Switzerland.

Sonnerup, B. U. Ö., I. Papamastorakis, G. Paschmann, and H. Luhr (1987), Magnetopause Properties from AMPTE/IRM Observations of the Convection Electric-Field - Method Development, J. Geophys. Res., 92(A11), 12137-12159.

Tan, B., Y. Lin, J. D. Perez, and X. Y. Wang (2011), Global-scale hybrid simulation of dayside magnetic reconnection under southward IMF: Structure and evolution of reconnection, J. Geophys. Res., 116, A02206, doi:10.1029/2010JA015580. 
ZHONG ET AL: : 3-D MAGNETIC FLUX ROPE STRUCTURE

Walker, R. J., and C. T. Russell (1985), Flux Transfer Events at the Jovian Magnetopause, J. Geophys. Res., 90(A8), 7397-7404.

Xiao, C. J., Z. Y. Pu, Z. W. Ma, S. Y. Fu, Z. Y. Huang, and Q. G. Zong (2004), Inferring of flux rope orientation with the minimum variance analysis technique, J. Geophys. Res., 109, A11218, doi:10.1029/2004JA010594.

Zhang, H., K. K. Khurana, M. G. Kivelson, V. Angelopoulos, Z. Y. Pu, Q. G. Zong, J. Liu, and X. Z. Zhou (2008), Modeling a force-free flux transfer event probed by multiple Time History of Events and Macroscale Interactions during Substorms (THEMIS) spacecraft, J. Geophys. Res., 113, A00C05, doi:10.1029/2008JA013451.

Zhang, H., M. G. Kivelson, V. Angelopoulos, K. K. Khurana, Z. Y. Pu, R. J. Walker, R. L. McPherron, T. S. Hsu, Q. G. Zong, and T. Phan (2012), Generation and properties of in vivo flux transfer events, J. Geophys. Res., 117, A05224, doi:10.1029/2011JA017166. 